International Journal of Pure and Applied Mathematics

Volume 86 No. 5 2013, 799-810

ISSN: 1311-8080 (printed version); ISSN: 1314-3395 (on-line version)

url: http://www.ijpam.eu

doi: http://dx.doi.org/10.12732/ijpam.v86i5.4

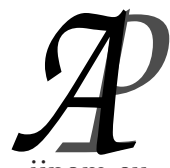

\title{
ON WEAKLY CONCIRCULAR SYMMETRIES OF THREE-DIMENSIONAL TRANS-SASAKIAN MANIFOLDS
}

\author{
Dakshayani A. Patil ${ }^{1}$, C.S. Bagewadi ${ }^{2}$ \\ ${ }^{1}$ Department of Mathematics \\ B.V.B. College of Engineering \& Technology \\ Hubli, 580 032, INDIA \\ ${ }^{2}$ Department of Mathematics \\ Kuvempu University \\ Jnana Sahyadri, 577 451, Shimoga, INDIA
}

\begin{abstract}
The purpose of this paper is to study weakly concircular symmetric and weakly concircular Ricci symmetric three-dimensional trans -Sasakian manifolds.
\end{abstract}

AMS Subject Classification: 53C15, 53C25

Key Words: weakly symmetric, weakly concircular symmetric, weakly Ricci symmetric, weakly concircular Ricci symmetric manifold, trans Sasakian manifold

\section{Introduction}

The notion of weakly symmetric manifolds was introduced by Tamassy and Binh[16]. A non flat Riemannian manifold $\left(M^{n}, g\right)(n>2)$ is called weakly symmetric if its curvature tensor $\mathrm{R}$ of type $(0,4)$ satisfies the condition

$$
\begin{aligned}
& \left(\nabla_{X} R\right)(Y, Z, U, V)=A(X) R(Y, Z, U, V)+B(Y) R(X, Z, U, V) \\
& +H(Z) R(Y, X, U, V)+D(U) R(Y, Z, X, V)+E(V) R(Y, Z, U, X)
\end{aligned}
$$

for all vector fields $X, Y, Z, U, V \in \chi\left(M^{n}\right)$, where $A, B, H, D$ and $E$ are

Received: $\quad$ March 21, 2013

(C) 2013 Academic Publications, Ltd.

${ }^{\S}$ Correspondence author 
1-forms(not simultaneously zero) and $\nabla$ denotes the operator of covariant differentiation with respect to the Riemannian metric $g$. The $1-$ forms are called the associated 1 -forms of the manifold and an $n$-dimensional manifold of this kind is denoted by $(W S)_{n}$. In 1999 De and Bandyopadhyay[4] studied a $(W S)_{n}$ and proved that in such a manifold the associated 1 -forms $B=H$ and $D=E$. Hence (1.1) reduces to the following form:

$$
\begin{aligned}
& \left(\nabla_{X} R\right)(Y, Z, U, V)=A(X) R(Y, Z, U, V)+B(Y) R(X, Z, U, V) \\
& +B(Z) R(Y, X, U, V)+D(U) R(Y, Z, X, V)+D(V) R(Y, Z, U, X)
\end{aligned}
$$

A transformation of an 3-dimensional Riemannian manifold $M$, which transforms every geodesic circle of $\mathrm{M}$ into a geodesic circle, is called a concircular transformation[18] and is defined by

$$
C(Y, Z) U=R(Y, Z) U-\frac{r}{6}[g(Z, U) Y-g(Y, U) Z]
$$

where $r$ is the scalar curvature of the manifold.

Recently Shaikh and Hui[14] introduced the notion of weakly concircular symmetric manifolds. A Riemannian manifold $\left(M^{n}, g\right)(n>2)$ is called weakly concircular symmetric manifold if its concircular curvature tensor $C$ satisfies the condition

$$
\begin{aligned}
& \left(\nabla_{X} C\right)(Y, Z, U)=A(X) C(Y, Z, U)+B(Y) C(X, Z, U) \\
& +H(Z) C(Y, X, U)+D(U) C(Y, Z, X)
\end{aligned}
$$

for all vector fields $X, Y, Z, U \in \chi\left(M^{3}\right)$, where $A, B, H$, and $D$ are 1-forms(not simultaneously zero) and 3 dimensional manifold of this kind is denoted by $(W C S)_{3}$. Also it is shown thatin a $(W C S)_{3}$ the associated 1 -forms $B=H$ , and hence the defining condition (1.4) of a $(W C S)_{3}$ reduces to the following form:

$$
\begin{aligned}
& \left(\nabla_{X} C\right)(Y, Z, U)=A(X) C(Y, Z, U)+B(Y) C(X, Z, U) \\
& +B(Z) C(Y, X, U)+D(U) C(Y, Z, X)
\end{aligned}
$$

$A, B$ and $D$ are 1 -forms(not simultaneously zero).

Again Tamassy and Binh[17] introduced the notion of weakly Ricci symmetric manifolds. A Riemannian manifold $\left(M^{n}, g\right)(n>2)$ is called weakly Ricci symmetric manifold if its Ricci tensor $S$ of type $(0,2)$ is not identically zero and satisfies the condition

$$
\left(\nabla_{X} S\right)(Y, Z)=A(X) S(Y, Z)+B(Y) S(X, Z)+D(Z) S(Y, X)
$$


where $A, B$ and $D$ are three non-zero 1 -forms, called the associated 1 -forms of the manifold, and $\nabla$ denotes the operator of covariant differentiation with respect to the metric $g$. Such an 3-dimensional manifold is denoted by $(W R S)_{3}$.

Let $e_{1}, e_{2}, e_{3}$ be an orthonormal basis of the tangent space at each point of the manifold and let

$$
P(Y, V)=\sum_{i=1}^{3} C\left(Y, e_{i}, e_{i}, V\right),
$$

then from (1.3), we get

$$
P(Y, V)=S(Y, V)-\frac{r}{3} g(Y, V) .
$$

The tensor $P$ is called the concircular Ricci symmetric tensor [5], which is a symmetric tensor of type $(0,2)$. In[5] De and Ghosh introduced the notion of weakly concircular Ricci symmetric manifolds. A Riemannian manifold $\left(M^{n}, g\right)(n>2)$ is called weakly concircular Ricci symmetric manifold [5]if its concircular Ricci tensor $P$ of type $(0,2)$ is not identically zero and satisfies the condition

$$
\left(\nabla_{X} P\right)(Y, Z)=A(X) P(Y, Z)+B(Y) P(X, Z)+D(Z) P(Y, X)
$$

where $A, B$ and $D$ are 1 -forms(not simultaneously zero).

In 1985, Oubina[11] introduced the notion of trans-Sasakian manifolds, which contains both the class of Sasakian and cosympletic structures, closely related to the locally conformal Kähler manifolds. Trans-Sasakian manifolds of type $(0,0),(\alpha, 0)$ and $(0, \beta)$ are the cosympletic, $\alpha$-Sasakian and $\beta$-Kenmotsu manifolds respectively. In particular, if $\alpha=1, \beta=0$ :and $\alpha=0, \beta=1$, then trans-Sasakian manifold reduces to a Sasakian and Kenmotsu manifolds respectively. Thus trans-Sasakian structures provide a large class of generalized quasi-Sasakian structures. The Local structure of trans-Sasakian manifolds of dimension $n \geq 5$ has been completely characterized by J. C. Marrero[9] . He proved that a trans-Sasakian manifold of dimension $n \geq 5$ is either cosymplectic or $\beta$-Kenmotsu or $\alpha$-Sasakian manifold. But when $n>3$ trans-Sasakian manifold does not exist In this paper we consider the three dimensional transSasakian manifold.

In 2002, Kim et al. [10] studied generalized Ricci recurrent trans-Sasakian manifolds. In [8] De and Tripathi studied Ricci semi-symmetric trans-Sasakian manifolds. Tamassy and Binh[17] studied weakly symmetric and weakly Ricci symmetric Sasakian manifolds and proved that in such a manifold the sum of the associated 1-forms vanishes everywhere. Again Özgür[12] studied weakly 
symmetric weakly Ricci symmetric Kenmotsu manifolds and proved that in such a manifold the sum of the associated 1-forms is zero everywhere and hence such a manifold does not exist unless the sum of the associated 1-forms is everywhere zero. Weakly symmetric and weakly Ricci symmetric properties for trans-Sasakian manifolds, Lorentzian $\alpha$-Sasakian manifolds were studied in [13], [1] and [15] respectively.

The object of the present paper is to study weakly concircular symmetric and weakly concircular Ricci symmetric trans-Sasakian manifolds. Section 2 deals with preliminaries of trans-Sasakian manifolds. In Section 3 of the paper we have obtianed all the 1 -forms of weakly concircular symmetric three dimensional trans-Sasakian manifold and hence such a structure exist. In the last section we study weakly concircular Ricci symmetric three dimensional trans-Sasakian manifolds and obtained all the 1 -forms of weakly concircular Ricci symmetric three dimensional trans-Sasakian manifold and consequently such a structure exist.

\section{Preliminaries}

A $(2 n+1)$-dimensional smooth manifold $M$ is said to be an almost contact metric manifold [2] if it admits a $(1,1)$ tensor field $\phi$, a vector field $\xi$, a 1 -form $\eta$, and a Riemannian metric $g$, which satisfy

$$
\begin{aligned}
& \phi \xi=0, \quad \eta(\phi X)=0, \quad \phi^{2} X=-X+\eta(X) \xi, \\
& g(\phi X, Y)=-g(X, \phi Y), \quad \eta(\xi)=1, \quad g(X, \xi)=\eta(X), \\
& g(\phi X, \phi Y)=g(X, Y)-\eta(X) \eta(Y)
\end{aligned}
$$

for all vector fields $\mathrm{X}, \mathrm{Y}$ on $\mathrm{M}$.

An almost contact metric manifold is said to be trans-Sasakian manifold[11] if $(M \times R, J, G)$ belongs to the class $W_{4}$ of the Hermitian manifolds, where $J$ is the almost complex structure on $M \times R$ defined by

$$
J\left(Z, f \frac{d}{d t}\right)=\left(\phi Z-f \xi, \eta(Z) \frac{d}{d t}\right)
$$

for any vector field $\mathrm{Z}$ on $\mathrm{M}$ and smooth function $f$ on $M \times R$ and $\mathrm{G}$ is product metric on $M \times R$. This may be stated by the condition[3]

$$
\left(\nabla_{X} \phi\right)(Y)=\alpha\{\{g(X, Y) \xi-\eta(Y) X\}+\beta\{g(\phi X, Y) \xi-\eta(Y) \phi X\},
$$

where $\alpha, \beta$ are smooth functions on $\mathrm{M}$ and such a structure is said to be the trans-Sasakian structure of type $(\alpha, \beta)$. from (2.4) it follows that

$$
\nabla_{X} \xi=-\alpha \phi X+\beta\{X-\eta(X) \xi\},
$$




$$
\left(\nabla_{X} \eta\right)(Y)=-\alpha g(\phi X, \phi Y) .
$$

In a trans-Sasakian manifold $M^{3}(\phi, \xi, \eta, g)$ the following relations hold [11]:

$$
\begin{aligned}
& R(X, Y) \xi=\left(\alpha^{2}-\beta^{2}\right)[\eta(Y) X-\eta(X) Y]-(X \alpha) \phi Y-(X \beta) \phi^{2}(Y) \\
&+\quad 2 \alpha \beta[\eta(Y) \phi X-\eta(X) \phi Y]+(Y \alpha) \phi X+(Y \beta) \phi^{2}(X), \\
& \eta(R(X, Y) Z)=\left(\alpha^{2}-\beta^{2}\right)[g(Y, Z) \eta(X)-g(X, Z) \eta(Y)] \\
&- 2 \alpha \beta[g(\phi X, Z) \eta(Y)-g(\phi Y, Z) \eta(X)] \\
&-(Y \alpha) g(\phi X, Z)-(X \beta)\{g(Y, Z)-\eta(Y) \eta(Z)\} \\
&+\quad(X \alpha) g(\phi Y, Z)-(Y \beta)\{g(X, Z)-\eta(X) \eta(Z)\}, \\
& \\
& S(X, \xi)=\left.2\left(\alpha^{2}-\beta^{2}\right)-(\xi \beta)\right] \eta(X)-((\phi X) \alpha)-(X \beta), \\
& R(\xi, X) \xi=\left(\alpha^{2}-\beta^{2}-\xi \beta\right)[\eta(X) \xi-X], \\
& S(\xi, \xi)=2\left(\alpha^{2}-\beta^{2}-\xi \beta\right), \\
&(\xi \alpha)+2 \alpha \beta=0 \\
& Q \xi=\left[2\left(\alpha^{2}-\beta^{2}\right)-(\xi \beta)\right] \xi+\phi(\operatorname{grad} \alpha)-(\operatorname{grad} \beta),
\end{aligned}
$$

where $R$ is the curvature tensor of type $(1,3)$ of the manifold and $Q$ is the symmetric endomorphism of the tangent space at each point of the manifold corresponding to the Ricci tensor $S$, that is , $g(Q X, Y)=S(X, Y)$ for any vector fields $X, Y$ on $M$.

\section{Weakly Concircular Symmetric Three-Dimensional Trans-Sasakian Manifolds}

Definition 1. A trans-Sasakian manifold $M^{3}(\phi, \xi, \eta, g)$ is said to be weakly concircular symmetric if its concircular curvature tensor $C$ satisfies (1.5).

Setting $Y=V=e_{i}$ in (1.5) and taking summation over $i, 1 \leq i \leq 3$, we get

$$
\begin{aligned}
& \left(\nabla_{X} S\right)(Z, U)-\frac{d r(X)}{3} g(Z, U)=A(X)\left[S(Z, U)-\frac{r}{3} g(Z, U)\right] \\
& +B(Z)\left[S(X, U)-\frac{r}{3} g(X, U)\right]+D(U)\left[S(X, Z)-\frac{r}{3} g(X, Z)\right] \\
& -\frac{r}{6}[\{B(X)+D(X)\} g(Z, U)-B(Z) g(X, U)-D(U) g(Z, X)] \\
& +B(R(X, Z) U)+D(R(X, U) Z)
\end{aligned}
$$


Putting $X=Z=U=\xi$ in (3.1) and then using (2.7) and (2.11) we obtain

$$
A(\xi)+B(\xi)+D(\xi)=\frac{\operatorname{grad} F \cdot \xi}{F}
$$

where $F=6\left(\alpha^{2}-\beta^{2}-\xi \beta\right)-r$

We can see that if $\operatorname{gradF}$ is orthogonal to $\xi$ then

$$
A(\xi)+B(\xi)+D(\xi)=0
$$

Since $A(X)=g(X, \rho), A(\xi)=B(\xi)=D(\xi)=g\left(\rho_{i} \xi_{i}\right)$

In view of (3.3) we obtain that $A(\xi)=B(\xi)=D(\xi)=0$.

If $\operatorname{grad} F$ and $\xi$ are not inclined orthogonal then $\operatorname{gradF} . \xi \neq 0$. Hence

$$
A(\xi)+B(\xi)+D(\xi) \neq 0
$$

that is $A(\xi)=B(\xi)=D(\xi) \neq 0$.

Theorem 1. In a weakly concircular trans-Sasakian manifold $M^{3}(\phi, \xi, \eta, g)$ the relation (3.2) holds.

Next substituting $X$ and $Z$ by $\xi$ in (3.1) and then using (2.9) and (2.12) we obtain

$$
\left(\nabla_{\xi} S\right)(\xi, U)=[A(\xi)+B(\xi)] S(U, \xi)+\left[\alpha^{2}-\beta^{2}-(\xi \beta)\right][D(U)+\eta(U) D(\xi)]
$$

Again we have

$$
\begin{aligned}
\left(\nabla_{\xi} S\right)(\xi, U)= & \nabla_{\xi} S(\xi, U)-S\left(\nabla_{\xi} \xi, U\right)-S\left(\xi, \nabla_{\xi} U\right) \\
= & {[2\{2 \alpha(\xi \alpha)-2 \beta(\xi \beta)\}-\xi(\xi \beta)] \eta(U) } \\
& -(U(\xi \beta))-(\phi U(\xi \alpha)),
\end{aligned}
$$

where (2.9) has been used. In view of (3.5) and (3.6) we obtain from (3.2) that

$$
\begin{aligned}
& D(U)=\frac{[12\{2 \alpha(\xi \alpha)-2 \beta(\xi \beta)\}-6 \xi(\xi \beta)] \eta(U)}{F}- \\
& \frac{6 U[\xi \beta]+6 \phi U[\xi \alpha]+2 \xi[r] \eta(u)}{F}-\left\{\frac{\xi[F]}{F^{2}}\right\} \\
& \left\{\left\{6\left[2\left(\alpha^{2}-\beta^{2}\right)-\xi \beta\right]-2 r\right\} \eta(U)-6\{U[\beta]+\phi U[\alpha]\}\right\}+ \\
& D(\xi)\left\{\frac{6\left\{\left(\alpha^{2}-\beta^{2}\right) \eta(U)-U[\beta]\right\}-6 \phi U[\alpha]-r \eta(U)}{F}\right\}
\end{aligned}
$$


for any vector field $U$.

If $\operatorname{gradF}$ and $\xi$ are orthogonal then by virtue of (3.2) and (3.3) we get

$$
\begin{aligned}
D(U)= & \frac{[12\{2 \alpha(\xi \alpha)-2 \beta(\xi \beta)\}-6 \xi(\xi \beta)] \eta(U)}{F} \\
& -\frac{6 U[\xi \beta]+6 \phi U[\xi \alpha]+2 \xi[r] \eta(u)}{F} \\
\neq & 0
\end{aligned}
$$

If $\operatorname{gradF}$ and $\xi$ are not orthogonal then by virtue of (3.4) we get $D(U) \neq 0$ Next setting $X=U=\xi$ in (3.1) and proceeding in a similar manner as above we get

$$
\begin{aligned}
& B(Z)=\frac{[12\{2 \alpha(\xi \alpha)-2 \beta(\xi \beta)\}-6 \xi(\xi \beta)] \eta(Z)}{F}- \\
& \frac{6 Z[\xi \beta]+6 \phi Z[\xi \alpha]+2 \xi[r] \eta(Z)}{F}-\left\{\frac{\xi[F]}{F^{2}}\right\} \\
& \left\{\left\{6\left[2\left(\alpha^{2}-\beta^{2}\right)-\xi \beta\right]-2 r\right\} \eta(Z)-6\{Z[\beta]+\phi Z[\alpha]\}\right\}+ \\
& B(\xi)\left\{\frac{6\left\{\left(\alpha^{2}-\beta^{2}\right) \eta(Z)-Z[\beta]\right\}-6 \phi Z[\alpha]-r \eta(Z)}{F}\right\}
\end{aligned}
$$

for any vector field $Z$

If $\operatorname{gradF}$ and $\xi$ are orthogonal then by virtue of (3.2) and (3.3) we get

$$
\begin{aligned}
B(Z)= & \frac{[12\{2 \alpha(\xi \alpha)-2 \beta(\xi \beta)\}-6 \xi(\xi \beta)] \eta(Z)}{F}- \\
& \frac{6 Z[\xi \beta]+6 \phi Z[\xi \alpha]+2 \xi[r] \eta(Z)}{F} \\
& 0
\end{aligned}
$$

If $\operatorname{grad} F$ and $\xi$ are not orthogonal then by virtue of $(3.4)$ we get $B(Z) \neq 0$

Again setting $Z=U=\xi$ in (3.1) we get

$$
\begin{aligned}
\left(\nabla_{\xi} S\right)(\xi, \xi)-\frac{d r(x)}{3} & =A(X)\left[S(\xi, \xi)-\frac{d r(x)}{3}\right]+B(R(X, \xi) \xi)+D(R(X, \xi) \xi) \\
& +[B(\xi)+D(\xi)]\left[S(X, \xi)-\frac{d r(x)}{3} \eta(X)\right] \\
& -\frac{r}{6}[B(X)+D(X)-B(\xi) \eta(X)-D(\xi) \eta(X)]
\end{aligned}
$$


Now we have

$$
\left(\nabla_{X} S\right)(\xi, \xi)=\nabla_{X} S(\xi, \xi)-2 S\left(\nabla_{X} \xi, \xi\right)
$$

which yields by using (2.5) and (2.9) that

$$
\begin{aligned}
\left(\nabla_{\xi} S\right)(\xi, \xi) & =2[2 \alpha(X \alpha)-2 \beta(X \beta)-(X(\xi \beta))] \\
& +2 \alpha[(X \alpha)-\eta(X)(\xi \alpha)-((\phi X) \beta)] \\
& +2 \beta[(\phi X) \alpha+\{(X \beta)-(\xi \beta) \eta(X)\}]
\end{aligned}
$$

using (2.10) (2.11) and (3.10) in $(3,9)$ we get

$$
\begin{aligned}
& A(X)=\frac{X(F+r)}{F}+\frac{6 \alpha\{(X \alpha)-\eta(X)(\xi \alpha)-(\phi X) \beta\}}{F}+ \\
& \frac{6 \beta[(\phi X)[\alpha]+\{X[\beta]-\xi \beta \eta(X)\}]-X[r]}{F}- \\
& \frac{[B(\xi)+D(\xi)]\left[3\left\{\left(\alpha^{2}-\beta^{2}\right) \eta(X)-X[\beta]\right\}-3 \phi X[\alpha]-\frac{r}{2} \eta(X)\right]}{F}- \\
& \frac{[B(X)+D(X)]\left[3\left(\alpha^{2}-\beta^{2}\right)-\xi \beta \frac{r}{2}\right]}{F}
\end{aligned}
$$

for any vector $X$,

If $\operatorname{grad} F$ and $\xi$ are orthogonal then by virtue of (3.2) and (3.3) we get

$$
\begin{aligned}
A(X)= & \frac{X(F+r)}{F}+\frac{6 \alpha\{(X \alpha)-\eta(X)(\xi \alpha)-(\phi X) \beta\}}{F} \\
& +\frac{6 \beta[(\phi X)[\alpha]+\{X[\beta]-\xi \beta \eta(X)\}]-X[r]}{F} \\
& -\frac{[B(X)+D(X)]\left[3\left(\alpha^{2}-\beta^{2}\right)-\xi \beta \frac{r}{2}\right]}{F} \\
\neq & 0
\end{aligned}
$$

If $\operatorname{gradF}$ and $\xi$ are not orthogonal then by virtue of (3.4) we get $A(X) \neq 0$ This leads the following:

Theorem 2. There exists no weakly Concircular symmetric trans-Sasakian manifold $M^{3}$, if $A+B+D$ is not everywhere zero. 


\section{Weakly Concircular Ricci Symmetric Three-Dimensional Trans-Sasakian Manifolds}

Definition 2. A trans-Sasakian manifold $M^{3}(\phi, \xi, \eta, g)$ is said to be weakly concircular Ricci symmetric if its concircular Ricci tensor $P$ of type $(0,2)$ satisfies $(1.9)$.

In view of (1.8), (1.9) yields

$$
\begin{aligned}
& \left(\nabla_{X} S\right)(Y, Z)-\frac{d r(X)}{3} g(Y, Z)=A(X)\left[S(Y, Z)-\frac{r}{3} g(Y, Z)\right] \\
& +B(Y)\left[S(X, Z)-\frac{r}{3} g(X, Z)\right]+D(Z)\left[S(X, Y)-\frac{r}{3} g(X, Y)\right]
\end{aligned}
$$

Setting $X=Y=Z=\xi \operatorname{in}(4.1)$, we get the relation (3.2) and hence we can state the following:

Theorem 3. In a weakly concircular Ricci symmetric trans-Sasakian manifold $M^{3}(\phi, \xi, \eta, g)$, the relation (3.2) holds.

Next, substituting $X$ and $Y$ by $\xi$ in (4.1) and using (2.9) and (3.2), we obtain

$$
\begin{aligned}
& D(Z)=\frac{\left\{6 \xi\left[\alpha^{2}-\beta^{2}\right]-(3) \xi[\xi \beta]\right\} \eta(Z)}{F}- \\
& \frac{3 Z \xi[\beta]+3 \phi Z[\xi \alpha]+\xi[r] \eta(Z)}{F}+ \\
& D(\xi) \frac{\left\{\left[6\left(\alpha^{2}-\beta^{2}\right)-3 \xi \beta-r\right] \eta(Z)-3 \phi Z[\alpha]-3 Z[\beta]\right\}}{F}- \\
& \left\{\frac{\xi[F]}{F^{2}}\right\}\left\{\left[2 n\left(\alpha^{2}-\beta^{2}\right)-(\xi \beta)\right] \eta(Z)-Z[\beta]-\phi Z[\alpha]-\frac{r}{3} \eta(Z)\right\}
\end{aligned}
$$

for any vector $Z$,

If $\operatorname{grad} F$ and $\xi$ are orthogonal then by virtue of (3.2) and (3.3) we get

$$
\begin{aligned}
D(Z)= & \frac{\left\{6 \xi\left[\alpha^{2}-\beta^{2}\right]-3 \xi[\xi \beta]\right\} \eta(Z)}{F} \\
& -\frac{3 Z \xi[\beta]+3 \phi Z[\xi \alpha]+\xi[r] \eta(Z)}{F} \\
\neq & 0
\end{aligned}
$$


If $\operatorname{grad} F$ and $\xi$ are not orthogonal then by virtue of $(3.4)$ we get $D(Z) \neq 0$ Again setting $X=Z=\xi$ in (4.1) and proceeding in a similar manner as above we get

$$
\begin{aligned}
& B(Y)=\frac{\left\{6 \xi\left[\alpha^{2}-\beta^{2}\right]-3 \xi[\xi \beta]\right\} \eta(Y)}{F}- \\
& \frac{3 Y \xi[\beta]+3 \phi Y[\xi \alpha]+\xi[r] \eta(Y)}{F}+ \\
& B(\xi) \frac{\left\{\left[6\left(\alpha^{2}-\beta^{2}\right)-3 \xi \beta-r\right] \eta(Y)-3 \phi Y[\alpha]-3 Y[\beta]\right\}}{F}- \\
& \left\{\frac{\xi[F]}{F^{2}}\right\}\left\{\left[2\left(\alpha^{2}-\beta^{2}\right)-(\xi \beta)\right] \eta(Y)-Y[\beta]-\phi Y[\alpha]-\frac{r}{3} \eta(Y)\right\}
\end{aligned}
$$

for any vector $Y$,

If $\operatorname{gradF}$ and $\xi$ are orthogonal then by virtue of (3.2) and (3.3) we get

$$
\begin{aligned}
B(Y)= & \frac{\left\{6 \xi\left[\alpha^{2}-\beta^{2}\right]-3 \xi[\xi \beta]\right\} \eta(Y)}{F} \\
& -\frac{3 Y \xi[\beta]+3 \phi Y[\xi \alpha]+\xi[r] \eta(Y)}{F} \\
\neq & 0
\end{aligned}
$$

If $\operatorname{grad} F$ and $\xi$ are not orthogonal then by virtue of (3.4) we get $B(Y) \neq 0$ Again putting $Y=Z=\xi$ in (4.1) and using (2.11) and (3.2), we get

$$
\begin{aligned}
& A(X)=\frac{X(F+r)}{F}+\frac{6 \alpha\{(X \alpha)-\eta(X)(\xi \alpha)-(\phi X) \beta\}}{F}+ \\
& \frac{6 \beta[(\phi X)[\alpha]+\{X[\beta]-\xi \beta \eta(X)\}]-X[r]}{F}+ \\
& A(\xi) \frac{\left\{\left[6\left(\alpha^{2}-\beta^{2}\right)-3 \xi \beta-r\right] \eta(X)-3 \phi X[\alpha]-3 X[\beta]\right\}}{F}- \\
& \left\{\frac{\xi[F]}{F^{2}}\right\}\left\{\left[2\left(\alpha^{2}-\beta^{2}\right)-(\xi \beta)\right] \eta(X)-X[\beta]-\phi X[\alpha]-\frac{r}{3} \eta(X)\right\}
\end{aligned}
$$

for any vector $X$,

If $\operatorname{grad} F$ and $\xi$ are orthogonal then by virtue of (3.2) and (3.3) we get

$$
A(X)=\frac{X(F+r)}{F}+\frac{6 \alpha\{(X \alpha)-\eta(X)(\xi \alpha)-(\phi X) \beta\}}{F}
$$




$$
\begin{array}{ll} 
& +\frac{6 \beta[(\phi X)[\alpha]+\{X[\beta]-\xi \beta \eta(X)\}]-X[r]}{F} \\
\neq & 0
\end{array}
$$

If $\operatorname{grad} F$ and $\xi$ are not orthogonal then by virtue of $(3.4)$ we get $A(X) \neq 0$ This leads the following:

Theorem 4. There exists no weakly Concircular Ricci symmetric transSasakian manifold $M^{3}$, if sum of the associated 1 -forms, $D, B$ and $A$ is not everywhere zero.

\section{References}

[1] C. S. Bagewadi, N. S. Basavarajappa, D. G. Prakasha, Venkatesh, On weakly symmetric and special weakly Ricci symmetric Lorentzian $\alpha$-Sasakian manifolds, Analele Unversitatii de Vest, Timisora, Seria Mathematica-Informatica, XLVI, No. 1 (2008), 3-12.

[2] D. E. Blair, Contact manifolds in Riemannian geometry, Lecture Notes in Math., 509, Springer-Verlag (1976).

[3] D. E. Blair, J. A. Oubina, Conformal and related changes of metric on the product of two almost contact metric manifolds, Publ. Math. Debrecen, $\mathbf{3 4}$ (1990), 199-207.

[4] U. C. De, S. Bandyopadhyay, On weakly symmetric Riemannian spaces, Publ. Math. Debrecen, 54 (1999), 377-381.

[5] U. C. De, G. C. Ghosh, On weakly concircular Ricci symmetric manifolds, South East Asian J. Math. and Math. Sci. , 3, No. 2 (2005), 9-15.

[6] U. C. De, T. Q. Binh, A. A. Shaikh, On weakly symmetric and weakly Ricci symmetric K-contact manifolds, Acta Math. Acad. Paedag. Nyigyhaz, 16 (2000), 65-71.

[7] U. C. De, A. A. Shaikh, S. Biswas, On weakly Ricci symmetric contact metric manifolds, Tensor N. S. , 28 (1994), 123-129.

[8] U. C. De, M. M. Tripathi, Ricci tensor in 3-dimensional trans-Sasakian manifolds, Kyungpook Math. J. , 43, No. 2 (2003), 247-225.

[9] J. C. Marrero, The local structure of trans-Sasakian manifolds, Ann. Mat. Pura Appl. , 162, No. 4 (1992), 77-86. 
[10] J. S. Kim, R. Prasad, M. M. Tripathi, On generalized Ricci-recurrenttransSasakian manifolds, J. Korean Math. Soc. , 39, No. 6 (2002), 953-961.

[11] J. A. Oubina, New class of almost contact metric manifolds, Publ. Math. Debrecen, 32 (1985), 187-193.

[12] C. Özgür, On weakly symmetric Kenmotsu manifolds, Diff. Geom. Dynam. Syst. , 8 (2006), 204-209.

[13] A. A. Shaikh, Shyamal Kumar Hui, On weak symmetries of trans-Sasakian manifolds, Proceedings of the Estonian Academy of Sciences, 58, No. 4 (2009), 213-223.

[14] A. A. Shaikh, Hui, On weakly concircular symmetric manifolds, Ann. Sti. Ale Univ. "Al. I. Cuza", Din Iasi, 1 (2009), 167-186.

[15] Shyamal Kumar Hui, On weak concircular symmetries of kenmotsu manifolds, Acta Universitatis Apulensis, 26 (2011), 129-136.

[16] L. Tamassy, T. Q. Binh, On weakly symmetric and weakly projective symmetric Rimannian manifolds, Coll. Math. Soc. J. Bolyai, 56 (1989), 663670 .

[17] L. Tamassy, T. Q. Binh, On weak symmetries of Einstein and Sasakian manifolds, Tensor N. S. , 53 (1993), 140-148.

[18] K. Yano, Concircular geometry I, Concircular transformations, Proc. Imp. Acad. Tokyo, 16 (1940), 195-200. 